\title{
Kinect Based Motion Capture for 3D Character Animation
}

\author{
Yaya Wihardi $^{1}$, Eka Fitrajaya Rahman ${ }^{2}$, Fadhil Farras H.N. ${ }^{3}$ \\ \{yayawihardi@upi.edu ${ }^{1}$, efitrajaya14@yahoo.co.id ${ }^{2}$, \\ fadhilfhn@student.upi.edu $\left.{ }^{3}\right\}$
}

Universitas Pendidikan Indonesia, Jl. Dr. Setiabudhi 229, Bandung, Indonesia ${ }^{1.2 .3}$

\begin{abstract}
In this research, we present a low-cost motion capture system for 3D character animation by utilizing an RGB-D sensor such as Kinect. The system captures the human motion and applies it on a 3D character. Animated 3D character recorded by a 3D animator environment and rendered as a part of animation production. To evaluate the timely execution of the system, we compare two rotation methods: Euler and Quaternion. The result shows that Quaternion more efficient than Euler.
\end{abstract}

Keywords: Motion capture, Animation, Kinect

\section{Introduction}

Motion capture is the process of recording a movement of objects by tracking the number of important points in three-dimensional space (3D) during a certain time. The results of this process usually translated into mathematical parameters for later use in various processes such as in 3D film production, animation, games [1], natural user interface[2], and robotics telecontrol [3], [4]. In the animation industry, the recorded motion is transferred to 3D character models to be rendered as an animation content.

To produce high-quality animation, we need a reliable sensor device and an effective 3D transformation process. Currently, a marker-based optical motion capture technology widely used in the animation industry. It requires the motion capture object to wear an item of special clothing that has been equipped with sensors or special markers. In general, this system requires proper lighting and a good reflection environment to produce a good result. This is quite difficult to install the device and the environment, and it also needs a high cost.

Kinect [5] is an alternative sensor that can be used in motion capture technology [6]. This device has a dual sensor that is an RGB camera and IR depth sensor so that it allows the user to capture the motion of humans without having to wear special clothes, and the installation process is quite easy. Moreover, the reliability level of this device is high [7] and the price is quite cheap [8]. However, the data captured by this device is only a coordinate of skeleton joints, so it cannot be used directly for 3D character animation.

This research presents a technique to transform output data from Kinect into transformation parameters that can be used for 3D character animation. The parameters are then transferred to the Blender 3D in a realtime manner through the Kinemator software that we have created. The animated movements of characters in Blender $3 \mathrm{D}$ are then recorded so that they can be rendered as an animation content. 


\section{Proposed System}

The proposed system consists of software and hardware that are interconnected as presented in Figure 1. Kinemator is the main data processing software that is connected to the Kinect by using a driver. It controls the kinect to retrieve data and terminate it in a certain situation. During the recording process, this software streams the motion data from kinect, and then convert it into transformation parameters. The results then sent to the Blender software through an addon that embedded in it. When the addon on Blender receives the data, this device applies the transformation parameters to predetermined 3D characters and records them. The recorded motion can be reviewed by the animator for editing and enhancement purpose. Finally, to produce the animation, we render the data by using Blender.

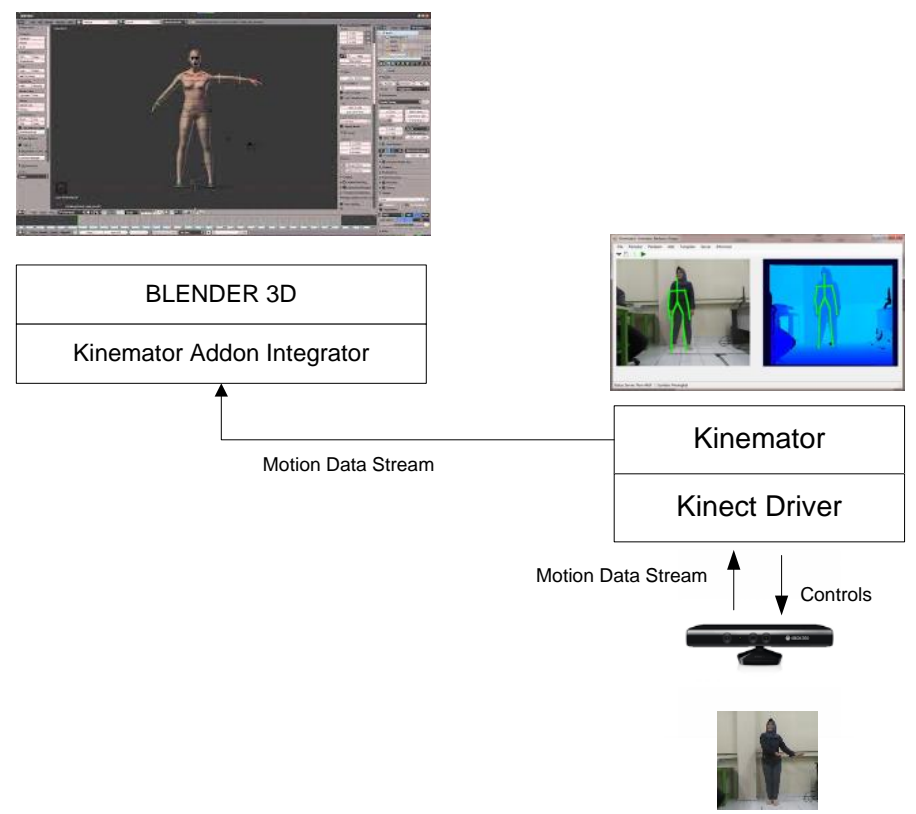

Fig. 1. The Proposed Motion Capture System.

\section{Motion Transfer}

Transfering the motion data to the 3D character can be done by applying the transformation process such as translation and rotation. The initial pose of the $3 \mathrm{D}$ character model is set to T-pose as shown in Figure 2. Captured pose/motion firstly computed to determine the translation and rotation parameter respect to the T-Pose. While for the rest frame, it's parameter calculated respect to the previous frame pose. Each translation and rotation parameter then applied to the $3 \mathrm{D}$ character model that has been determined in the Blender software.

\subsection{Joints Mapping and Transformation}


To transfer the motion, we need to map the captured joints skeleton to the $3 \mathrm{D}$ character joints skeleton. There are 15 joints skeleton captured by the system as shown in Table 1. Each joint representative a center of rotation of certain bone and its child. This joints mapping schema shown in Figure 2.

Motion transfer only translates the root join (No. 0, See in Figure 2) in the world coordinate system, while the rest only rotate by respect to its parent. In this research, we use two kinds of rotation techniques: Euler and Quaternion.

Table 1. Captured Joints.

\begin{tabular}{clc}
\hline $\begin{array}{c}\text { Joint } \\
\text { Number }\end{array}$ & \multicolumn{1}{c}{$\begin{array}{c}\text { Joint } \\
\text { Name }\end{array}$} & $\begin{array}{c}\text { Joint } \\
\text { Parent }\end{array}$ \\
\hline 0 & Torso & - \\
1 & Neck & 0 \\
2 & Head & 1 \\
3 & Left Shoulder & 1 \\
4 & Left Elbow & 3 \\
5 & Left Hand & 4 \\
6 & Right Shoulder & 1 \\
7 & Right Elbow & 6 \\
8 & Right Hand & 7 \\
9 & Left Hip & 0 \\
10 & Left Knee & 9 \\
11 & Left Foot & 10 \\
12 & Right Hip & 0 \\
13 & Right Knee & 12 \\
14 & Right Foot & 13 \\
& & \\
\hline
\end{tabular}
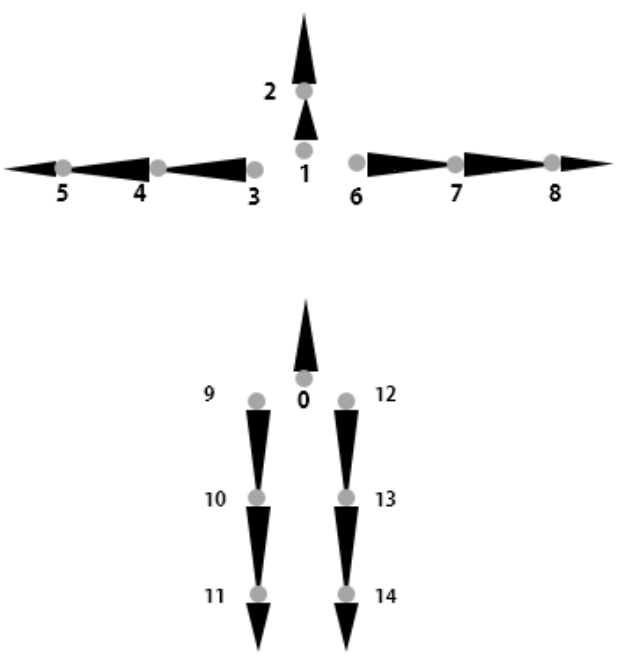

Fig. 2. T-Pose Skeleton and Joints.

\subsection{Blender Integration}


All calculated transformation parameters then transferred to the Blender by using embedded Blender-addon. Kinemator and the embedded-addon communicate through a serial socket on a certain port. The embedded-addon not only applying transformation parameters to the 3D Character as shown in Figure 3 but also record the rotation time for analysis purpose.

\section{Results and discussion}

To evaluate the performance of the proposed system, we apply the transformation by using 30 frames of motion data. Based on the experiment, we obtain rotation time as shown in Table 2. The average of rotation time for Quaternion and Euler respectively are 4.7 milliseconds and 5.73 milliseconds. The difference of rotation time only 1 millisecond, but it is significant since the number of the frame in an animation is very much and it may be repeated.

Table 2. Rotation Time.

\begin{tabular}{|c|c|c|}
\hline Frame & $\begin{array}{c}\text { Quaternion } \\
\text { (milisecond) }\end{array}$ & $\begin{array}{c}\text { Euler } \\
\text { (milisecond) }\end{array}$ \\
\hline 1 & 4 & 4 \\
\hline 2 & 7 & 6 \\
\hline 3 & 5 & 9 \\
\hline 4 & 5 & 7 \\
\hline 5 & 4 & 5 \\
\hline 6 & 5 & 9 \\
\hline 7 & 6 & 8 \\
\hline 8 & 4 & 7 \\
\hline 9 & 2 & 4 \\
\hline 10 & 7 & 6 \\
\hline 11 & 4 & 8 \\
\hline 12 & 2 & 5 \\
\hline 13 & 7 & 8 \\
\hline 14 & 7 & 5 \\
\hline 15 & 7 & 6 \\
\hline 16 & 7 & 3 \\
\hline 17 & 7 & 6 \\
\hline 18 & 2 & 9 \\
\hline 19 & 3 & 5 \\
\hline 20 & 3 & 4 \\
\hline 21 & 6 & 6 \\
\hline 22 & 6 & 4 \\
\hline 23 & 7 & 7 \\
\hline 24 & 3 & 2 \\
\hline 25 & 2 & 4 \\
\hline 26 & 5 & 6 \\
\hline 27 & 4 & 5 \\
\hline 28 & 4 & 9 \\
\hline 29 & 3 & 2 \\
\hline 30 & 3 & 3 \\
\hline Average & 4.7 & 5.73 \\
\hline
\end{tabular}



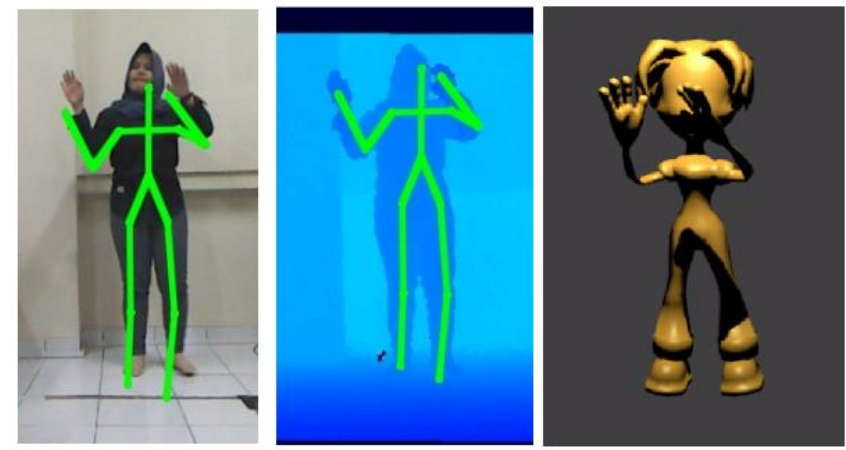

Fig. 3. Transferred Motion to a 3D Character.

\section{Conclusion}

It is visible and reliable to utilize kinect-based motion capture in 3D character animation pipeline. The installation process and environment setting are quite easy and need a low cost. In the term of transformation methods, Quaternion takes rotation time more eficient than Euler methods, so that it can be the best choice.

\section{References}

[1] X. Tong, P. Xu, and X. Yan, "Research on Skeleton Animation Motion Data Based on Kinect," in 2012 Fifth International Symposium on Computational Intelligence and Design, 2012, pp. 347-350.

[2] W.-S. Yu and K.-Y. Huang, "Implementation of media player simulator using Kinect sensors," in 2017 International Conference on System Science and Engineering (ICSSE), 2017, pp. 204-209.

[3] C. Vongchumyen, C. Bamrung, W. Kamintra, and A. Watcharapupong, "Teleoperation of Humanoid Robot by Motion Capturing Using KINECT," in 2018 International Conference on Engineering, Applied Sciences, and Technology (ICEAST), 2018, pp. 1-4.

[4] C. Li, C. Yang, P. Liang, A. Cangelosi, and J. Wan, "Development of Kinect based teleoperation of Nao robot," in 2016 International Conference on Advanced Robotics and Mechatronics (ICARM), 2016, pp. 133-138.

[5] Microsoft, "Kinect." [Online]. Available: http://www.xbox.com/kinect.

[6] N. H. B. Ismail and S. N. B. Basah, "The applications of Microsoft Kinect for human motion capture and analysis : A review," in 2015 2nd International Conference on Biomedical Engineering (ICoBE), 2015, pp. 1-4.

[7] George R, M. An, Feng X, and S. Sa, "Free Communications, Poster Presentations: Instrumentation Reliability/Validity Reliability of Kinect-Based Markerless Motion Capture During Lower Extremity Tasks,” 2017.

[8] F. Schlagenhauf, P. P. Sahoo, and W. Singhose, "Comparison of single-kinect and dual-kinect motion capture of upper-body joint tracking," in 2017 11th Asian Control Conference (ASCC), 2017, pp. 256-261. 